\title{
The Effect of Acute and Chronic Exposure to Hypobaric Hypoxia on Loaded Squat Jump Performance
}

\author{
by \\ Amador García-Ramos ${ }^{1}$, Paulino Padial ${ }^{1}$, Blanca De la Fuente ${ }^{2}$, \\ Javier Argüelles-Cienfuegos², Juan Bonitch-Góngora ${ }^{1}$, Belén Feriche ${ }^{1}$
}

\begin{abstract}
The present study aimed (1) to compare loaded squat jump performance after an acute and chronic exposure to a moderate natural altitude between normoxia and hypobaric hypoxia conditions, and (2) to analyze the effect of an altitude training camp on loaded jump squat development. Sixteen male swimmers (17.1 \pm 0.8 years) took part in a 17day training camp at a natural moderate altitude. They were randomly tested in counterbalanced order on days 1 and 3 in normoxia and hypoxia (pretest) and on days 15 and 17 again in normoxia and hypoxia (posttest). The peak velocity reached with loads equivalent to $25 \%, 50 \%, 75 \%$ and $100 \%$ of swimmers' pretest body weight in the loaded squat jump exercise was the dependent variable analyzed. An overall increase in peak velocity during the test performed in hypoxia of $6.5 \%$ in pretest $(p<0.001, E S=0.98)$ and $4.5 \%$ in posttest $(p<0.001, E S=0.81)$ was observed. An overall increment in peak velocity of $4.0 \%$ considering the data for normoxia tests $(p<0.001, E S=0.61)$ and $2.1 \%$ considering the data for hypoxia tests $(p=0.008, E S=0.36)$ was achieved after the altitude training camp. These results highlight the beneficial effects of hypobaric hypoxia on jump performance after short and longer term exposure to a natural moderate altitude. The increase in loaded squat jump performance following the 17-day training camp suggests that altitude training could constitute a favorable stimulus in explosive strength.
\end{abstract}

Key words: altitude training, lower-limb muscular power, linear velocity transducer, swimmers.

\section{Introduction}

The hypobaric hypoxic conditions induced by increasing the altitude have profound effects on athletic performance (Kenney et al., 2012; Levine et al., 2008). Physical factors due to the reduction in the barometric pressure (hypobaric) and/or physiological factors arising from the reduced partial pressure of oxygen in the inspired air (hypoxia) may be responsible for the changes in physical performance (Levine et al., 2008). As the altitude increases, aerobic performance and maximal oxygen uptake are impaired due to the hypoxic environment (Fulco et al., 1998; Wehrlin and Hallen, 2006). In contrast to long-duration activities at which the aerobic metabolism is paramount, for high-intensity activities lasting less than $1 \mathrm{~min}$, the predominant energy source is phosphorylation and nonoxidative production of ATP (di Prampero, 2003). Therefore, it is not expected that such activities are compromised by the decrease in oxygen availability (Feriche et al., 2014; Levine et al., 2008).

Certainly, an acute ascent in the altitude has been related to an improvement in the performance of explosive actions (Kenney et al., 2012). The multiple world records broken in short events (sprinting and jumping) during the 1968 Mexico Olympics held at $2240 \mathrm{~m}$, as well as other

1 - Department of Physical Education and Sport, Faculty of Sport Sciences, University of Granada.

2 - High performance Center of Sierra Nevada, Spanish Sport Council. 
positive results reported in scientific research into explosive actions performed at the altitude support this assertion (Hahn and Gore, 2001; Levine et al., 2008). Reduction in the external resistance to the movement due to the decrease in air density at the altitude $(\sim 3 \%$ reduction for each $305 \mathrm{~m}$ rise [Levine et al., 2008]) has been proposed as the principle explanation for these results (Hahn and Gore, 2001; Kenney et al., 2012; Levine et al., 2008; Peronnet et al., 1991). Nevertheless, different physiological factors such as additional recruitment of fast twitch muscle fibers (Schoenfeld, 2013) or the increased activity of the sympathetic nervous system (Hainsworth et al., 2007) could also be implicated.

In contrast, chronic exposure to the altitude has been related to a deterioration in lean mass (Deldicque and Francaux, 2013; Mizuno et al., 2008) and its functional capacity (Felici et al., 2001; Ferretti et al., 1990; Narici and Kayser, 1995; Raguso et al., 2004). The effect of hypoxia itself on the protein metabolism (Deldicque and Francaux, 2013; Etheridge et al., 2011), an insufficient energy intake (Aeberli et al., 2013; Fulco et al., 2002) or a reduced training stimulus (Feriche et al., 2014; Hoppeler and Desplanches, 1992) have also been identified as possible explanations for this impairment. However, the studies that have found adverse effects of chronic hypoxia on the muscle size and strength/power adaptations were conducted at a high altitude ( $>5000 \mathrm{~m}$ asl). Therefore, it is necessary to study the effect of chronic exposure to a moderate natural altitude (2000-2500 m asl) on muscle power adaptations.

An increased velocity against the same absolute load has been described during traditional resistance training exercises such as the bench press (Feriche et al., 2014) and half-squat (Chirosa et al., 2006) following a sudden ascent (within 1-5 hours) to a moderate altitude $(2320 \mathrm{~m}$ asl) from normoxic conditions. However, whether this enhanced performance could be maintained after a longer period at the altitude is unknown. Similarly, there is a shortage of knowledge about the development in explosive strength performance after a training camp at a moderate altitude. Therefore, the aims of the present study were (1) to compare loaded squat jump performance after an acute (1-3 days) and chronic (15-17 days) exposure to a moderate natural altitude between normoxia and hypobaric hypoxia conditions, and (2) to analyze the effect of an altitude training camp on loaded jump squat development.

\section{Material and Methods}

\section{Participants}

Sixteen male swimmers from the Spanish Junior National Team (age $17.1 \pm 0.8$ years, body height $1.81 \pm 0.07 \mathrm{~m}$, body mass $73.9 \pm 7.8 \mathrm{~kg}$ ) volunteered to participate in this study. Swimmers were in their competitive period and had participated in national and international competitions for at least one year prior to the beginning of the study. All participants were informed of the procedures to be utilized and signed a written informed consent form prior to investigation. For swimmers under 18 years old, consent was obtained from their legal guardians. The study protocol adhered to the tenets of the Declaration of Helsinki and was approved by the University of Granada Institutional Review Board. Study design

A repeated-measures design was used to investigate the effect of an acute and chronic exposure to hypobaric hypoxia on loaded squat jump performance. The participants were assessed and monitored over a 17-day period at the High Performance Centre of Sierra Nevada (Spain), located at $2320 \mathrm{~m}$ asl. During this period, swimmers were tested four times, twice in normoxia (690 m asl) and twice in hypobaric hypoxia $(2320 \mathrm{~m}$ asl). The swimmers were randomly tested in counterbalanced order on days 1 and 3 (acute exposure) in both normoxia $\left(\mathrm{N}_{1}\right)$ and hypoxia $\left(\mathrm{H}_{1}\right)$ and again on days 15 and 17 (chronic exposure) in normoxia $\left(\mathrm{N}_{2}\right)$ and hypoxia $\left(\mathrm{H}_{2}\right)$. The individual load-velocity relationships with loads equivalent to $25 \%, 50 \%$, $75 \%$ and $100 \%$ of swimmers' pretest body weight (BW) in the loaded squat jump exercise was determined on each day of testing. Peak velocity (PV) was collected with a linear velocity transducer (T-Force System) to compare the acute effect of altitude exposure $\left(\mathrm{N}_{1}\right.$ vs. $\mathrm{H}_{1}$ and $\mathrm{N}_{2}$ vs. $\mathrm{H}_{2}$ ) as well as the adaptation after the training camp ( $\mathrm{N}_{1}$ vs. $\mathrm{N}_{2}$ and $\mathrm{H}_{1}$ vs. $\mathrm{H}_{2}$ ). An overview of the experimental procedure is depicted in Figure 1.

\section{Procedures}

After determining the athletes' body height (Seca 202, Seca Ltd., Hamburg, Germany) 
and mass (Tanita BC 418 segmental, Tanita corporation, Tokyo, Japan), swimmers completed a standardized warm-up based on jogging, joint mobility, dynamic stretching, six jumps without additional weight and one set of five jumps lifting $17 \mathrm{~kg}$ in the assessed exercise. Subjects then performed an incremental loading test in the loaded squat jump exercise. The loads used were $25 \%, 50 \%, 75 \%$ and $100 \%$ of the swimmers' pretest BW. Since the weight of the unloaded Smith machine bar was $17 \mathrm{~kg}$, the swimmers with body mass $<68 \mathrm{~kg}$ performed their first set with a relative intensity a bit higher than $25 \%$ of BW. The absolute loads used during the test were $19.0 \pm 1.8$ $\mathrm{kg}(25.4 \pm 0.7 \% \mathrm{BW}), 37.5 \pm 3.9 \mathrm{~kg}(50.0 \pm 0.3 \% \mathrm{BW})$, $56.1 \pm 5.8 \mathrm{~kg}(74.9 \pm 0.3 \% \mathrm{BW})$ and $74.4 \pm 7.8 \mathrm{~kg}$ $(100.1 \pm 0.3 \% \mathrm{BW})$. The same absolute loads for each subject were used in all tests, allowing the assessment of the load-velocity relationship in identical loading conditions (Hansen et al., 2011). Two repetitions were performed with each load. Recovery time was 1 min between attempts with the same load and $5 \mathrm{~min}$ between the attempts with different loads.

The movement commenced from a standing position with the knees and hips fully extended, feet approximately shoulder-width apart and the barbell resting across the back at the level of the acromion. The swimmers then slowly assumed a squatted position until the back of the thigh touched a rod on a tripod set at a knee angle of $90^{\circ}$ previously measured with a manual goniometer (Bazuelo-Ruiz et al., 2015). The subjects were instructed to maintain this position for two seconds before performing a purely concentric action in order to jump as high as possible (Markovic and Jaric, 2007). Movements such as countermovement or throwing the bar over the shoulders were not allowed. If any of these movements were observed, the jump was repeated after a corresponding rest period.

All the tests were performed with a Smith machine (Technogym, Barcelona, Spain). A dynamic measurement system (T-Force System; Ergotech, Murcia, Spain) validated by SánchezMedina and González-Badillo (2011) was fixed perpendicularly to the bar to record directly its vertical instantaneous velocity at a frequency of $1000 \mathrm{~Hz}$. Peak velocity (PV) was defined as the maximum instantaneous velocity value attained during the concentric phase of each repetition.
Peak velocity was used since this variable had shown to be closely related to vertical jump performance (i.e., jump height) (García-Ramos et al., 2015). Only the repetition with the highest PV at each load was considered for further analysis. In addition, an overall PV value for each subject was calculated by averaging the PV values of each individual load.

\section{Training prescription}

The swimmers completed the training programs prescribed by their coaches and the training load was monitored in training diaries. One coach was responsible for filling in the training diary of each swimmer. On average, swimmers performed 25 pool sessions (mean \pm standard deviations [SD]; duration: $119.8 \pm 10.8$ min, CR-10 RPE (Borg et al., 1985): $7.3 \pm 0.9$, and distance: $6696 \pm 644 \mathrm{~m}$ ) and 10 dry land sessions (4 sessions of circuit-training and 6 of strengthpower training). The lower limb exercises performed by the swimmers were: the half-squat (3-4 sets of 6-8 repetitions with $70-90 \%$ of $\mathrm{BW}$ and fast speed) and the lunge (3-4 sets of 6-12 repetitions with $30 \%$ of BW and moderate speed). Prior to the study outset, swimmers had been following similar resistance training routines as the one carried out in the present study. Additionally, they were requested to include the exercises employed during the altitude training camp as part of their training schedule at least 2 months before the beginning of the study.

\section{Statistical analysis}

Data are presented as mean \pm SD. A threeway (test [pretest and posttest] $\times$ condition [normoxia and hypoxia] $\times$ load [25\%, $50 \%, 75 \%$, and $100 \%$ of BW]) repeated measures ANOVA was used to examine peak barbell velocity during the different tests performed. When a significant $\mathrm{F}$ value was achieved, pairwise differences between means were identified using Bonferroni post hoc procedures. The magnitude of the differences was expressed as a standardized mean difference (Cohen's d effect size; ES). The criteria to interpret the magnitude of the ES were as follows: $<0.2=$ trivial, $0.2-0.6=$ small, $0.6-1.2=$ moderate, $1.2-2.0$ $=$ large, and $>2=$ very large (Hopkins et al., 2009). Test-retest absolute reliability was measured by the standard error of measurement (SEM) which was expressed in relative terms through the coefficient of variation (CV), whereas relative reliability was assessed by the intraclass 
correlation coefficients (ICC) calculated with the two-way random effects model. The reliability analysis was done between the two repetitions performed with each load in the pretest for each specific environmental condition (normoxia or hypoxia). Given that the order of the tests in normoxia and hypoxia was counterbalanced, the learning effect in the loaded squat jump exercise was assessed comparing the data from test 1 (50\% of the data came from the normoxia test and remaining $50 \%$ from the hypoxia test) and test 2 , in both pretest and posttest. The T-test for paired data and ES were used to compare test 1 and test 2 in pretest and posttest. All statistical tests were performed using the software package SPSS (version 20.0: SPSS, Inc., Chicago, IL, USA). The level of significance was set at $p<0.05$ and the confidence interval at $95 \%$ was indicated when appropriate $(95 \% \mathrm{CI})$.

\section{Results}

The three-way repeated measures ANOVA revealed significant main effects for the test $\left(\mathrm{F}[1,15]=33.6, p<0.001, \eta_{p}^{2}=0.691\right)$, condition $\left(\mathrm{F}[1,15]=135.1, p<0.001, \eta_{p}^{2}=0.900\right)$ and load $\left(\mathrm{F}[1.4,21.7]=1888.6, p<0.001, \eta_{p}^{2}=\right.$ 0.991). However, neither of the interactions reached statistical significance: test $\mathrm{x}$ condition $\left(\mathrm{F}[1,15]=2.32, p=0.149, \eta_{p}^{2}=0.134\right)$, test $\mathrm{x}$ load $\left(\mathrm{F}[3,45]=1.99, p=0.129, \eta_{p}^{2}=0.117\right)$, condition $\mathrm{x}$ load $\left(\mathrm{F}[3,45]=1.27, p=0.296, \eta_{p}^{2}=0.078\right)$ and test $\mathrm{x}$ condition $\mathrm{x}$ load $\left(\mathrm{F}[3,45]=0.06, p=0.983, \eta_{p}^{2}=\right.$ $0.004)$.

Table 1 summarizes PV descriptive values for all conditions tested in the present study. An overall increase in the load-velocity relationship of $3.1 \%(95 \% \mathrm{CI}=1.9-4.2 \%)$ in the posttest $(2.128 \pm$ $\left.0.115 \mathrm{~m} \cdot \mathrm{s}^{-1}\right)$ compared to the pretest $(2.065 \pm 0.126$ $\left.\mathrm{m} \cdot \mathrm{s}^{-1}\right)$ was observed $(p<0.001)$. A $5.5 \%(95 \% \mathrm{CI}=$ 4.5-6.5\%) increase in PV during the test conducted in hypoxia $\left(2.153 \pm 0.120 \mathrm{~m} \cdot \mathrm{s}^{-1}\right)$ compared to normoxia $\left(2.041 \pm 0.120 \mathrm{~m} \cdot \mathrm{s}^{-1}\right)$ was reached $(p<$ 0.001).

Normoxia 1 vs. Hypoxia $1 / /$ Normoxia 2 vs. Hypoxia 2

Higher PV values were reached during the tests conducted in hypoxia in both pretest and posttest with the four loads analyzed (Figure 2).

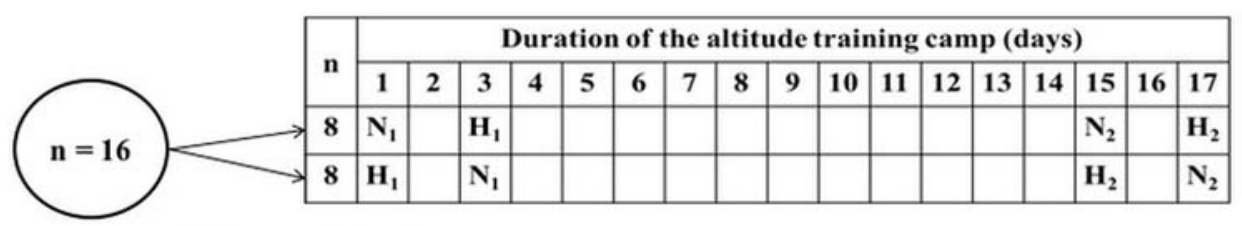

Environmental conditions

Hypobaric hypoxia (2320 asl, H) Normoxia (690 asl, N)
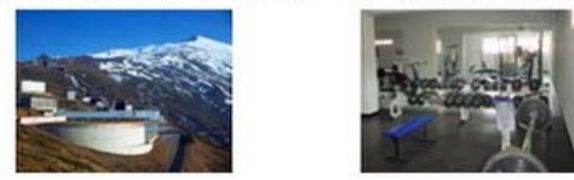

4 loads

$25 \%, 50 \%, 75 \%$ and $100 \%$ body weight

Figure 1

Overview of the experimental procedure used in the study.

$N_{1}$, pretest conducted in normoxia; $H_{1}$, pretest conducted in hypoxia;

$\mathrm{N}_{2}$, posttest conducted in normoxia;

$\mathrm{H}_{2}$, posttest conducted in hypoxia; $n$, number of subjects 
Table 1

Peak velocity values $\left(\mathrm{m} \cdot \mathrm{s}^{-1}\right)$ for the different conditions tested

\begin{tabular}{ccccc}
\hline \multirow{2}{*}{$\begin{array}{c}\text { Load } \\
\text { (\% body weight) }\end{array}$} & \multicolumn{2}{c}{ Pretest } & \multicolumn{2}{c}{ Posttest } \\
\cline { 2 - 5 } & Normoxia & Hypoxia & Normoxia & Hypoxia \\
\hline $25 \%$ & $2.463 \pm 0.17$ & $2.570 \pm 0.17$ & $2.539 \pm 0.12$ & $2.615 \pm 0.15$ \\
$50 \%$ & $2.104 \pm 0.16$ & $2.237 \pm 0.13$ & $2.205 \pm 0.13$ & $2.295 \pm 0.14$ \\
$75 \%$ & $1.843 \pm 0.13$ & $1.985 \pm 0.13$ & $1.908 \pm 0.12$ & $2.012 \pm 0.11$ \\
$100 \%$ & $1.591 \pm 0.11$ & $1.728 \pm 0.11$ & $1.673 \pm 0.10$ & $1.781 \pm 0.09$ \\
\hline
\end{tabular}

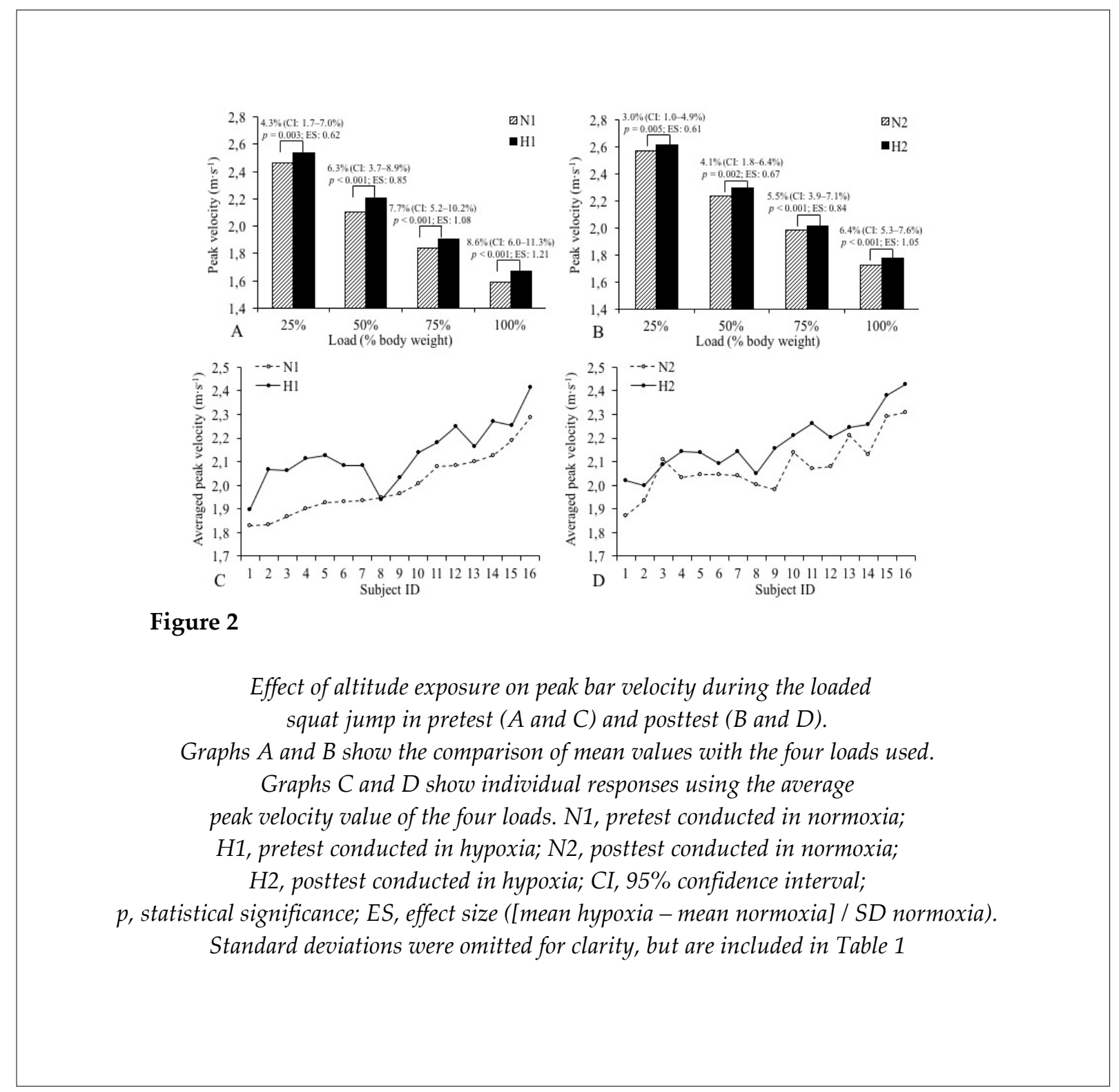



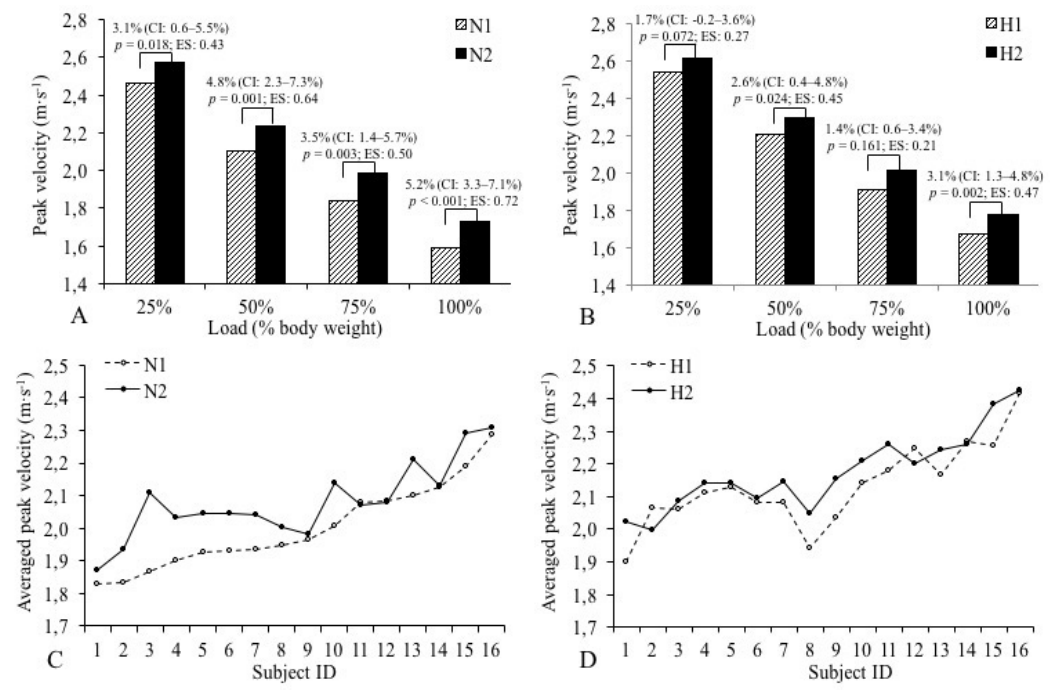

Figure 3

Chronic effects of the altitude training camp on peak bar velocity during the loaded jump squat.

Graphs $A$ and $B$ show the comparison of mean values with the four loads used.

Graphs $C$ and $D$ show individual responses using the average peak

velocity value of the four loads. N1, pretest conducted in normoxia;

$\mathrm{N} 2$, posttest conducted in normoxia; H1, pretest conducted in hypoxia;

$H 2$, posttest conducted in hypoxia; CI, 95\% confidence interval; $p$, statistical significance;

$E S$, effect size ([mean posttest - mean pretest] / SD pretest).

Standard deviations were omitted for clarity but are included in Table 1.

Table 2

Peak velocity $\left(\mathrm{m} \cdot \mathrm{s}^{-1}\right)$ comparisons between test 1 and test 2 in pre- and posttest

\begin{tabular}{ccccc}
\hline \multirow{2}{*}{$\begin{array}{c}\text { Load } \\
\text { (\% body weight })\end{array}$} & \multicolumn{2}{c}{ Pretest } & \multicolumn{2}{c}{ Posttest } \\
\cline { 2 - 5 } & Test 1 & Test 2 & Test 1 & Test 2 \\
\hline $25 \%$ & $2.516 \pm 0.17$ & $2.517 \pm 0.19$ & $2.564 \pm 0.15$ & $2.589 \pm 0.14$ \\
$50 \%$ & ES $=0.003$ & ES $=0.063$ \\
& $2.163 \pm 0.16$ & $2.179 \pm 0.16$ & $2.232 \pm 0.16$ & $2.268 \pm 0.13$ \\
$75 \%$ & ES $=0.034$ & ES $=0.082$ \\
& $1.906 \pm 0.15$ & $1.921 \pm 0.15$ & $1.947 \pm 0.15$ & $1.973 \pm 0.11$ \\
$100 \%$ & ES $=0.027$ & ES $=0.052$ \\
& $1.643 \pm 0.14$ & $1.676 \pm 0.12$ & $1.721 \pm 0.13$ & $1.733 \pm 0.09$ \\
& ES $=0.054$ & ES $=0.021$ \\
Overall & $2.057 \pm 0.14$ & $2.073 \pm 0.15$ & $2.116 \pm 0.14$ & $2.141 \pm 0.11$ \\
& ES $=0.033$ & ES $=0.053$ \\
\hline
\end{tabular}

Overall, data obtained averaging the four loads; ES, effect size ([mean Test 2 - mean Test 1] / SD both). 
An overall increase in PV during the test performed in hypoxia of $6.5 \%$ in the pretest $(95 \%$ $\mathrm{CI}=4.7-8.2 \%, p<0.001, \mathrm{ES}=0.98)$ and $4.5 \%$ in the posttest $(95 \% \mathrm{CI}=3.2-5.9 \%, p<0.001$, ES $=0.81)$ was observed. All swimmers, apart from subject 8 in the pretest $\left(\mathrm{N}_{1}=1.948 \mathrm{~m} \cdot \mathrm{s}^{-1}\right.$ and $\left.\mathrm{H}_{1}=1.941 \mathrm{~m} \cdot \mathrm{s}^{-1}\right)$ and subject 3 in the posttest $\left(\mathrm{N}_{2}=2.108 \mathrm{~m} \cdot \mathrm{s}^{-1}\right.$ and $\mathrm{H}_{2}=2.086 \mathrm{~m} \cdot \mathrm{s}^{-1}$ ), showed higher PV values during the tests conducted in hypoxia (Figure 2).

Normoxia 1 vs. Normoxia 2 // Hypoxia 1 vs. Hypoxia 2

Significant increases in PV were observed after the training period with the four loads analyzed (Figure 3). An overall increment of $4.0 \%$ $(95 \%$ CI $=2.3-5.8 \%, p<0.001, \quad$ ES $=0.61)$ considering the data for normoxia tests and $2.1 \%$ $(95 \%$ CI $=0.6-3.7 \%, p=0.008, \quad$ ES $=0.36)$ considering the data for hypoxia tests was achieved. Analysis of the data recorded in normoxia showed that $75 \%$ of the swimmers $(\mathrm{n}=$ 12) improved PV performance by more than $1 \%$, whereas changes in the remaining $25 \%$ of the subjects $(n=4)$ were between $+1 \%$ and $-1 \%$. Considering the data recorded in hypoxia, $62.5 \%$ of the swimmers $(\mathrm{n}=10)$ improved PV performance by more than $1 \%, 25 \%(\mathrm{n}=4)$ were between $+1 \%$ and $-1 \%$, and $12.5 \%(n=2)$ showed a reduction in $\mathrm{PV}$ performance by more than $1 \%$.

\section{Learning effects}

High test-retest reliability for PV values was observed in the pretest at $25 \% \mathrm{BW}(\mathrm{CV}: 2.5 \%$, ICC: 0.89 [0.79-0.94]), 50\% BW (CV: $2.1 \%$, ICC: 0.92 [0.84-0.96]), 75\% BW (CV: 3.1\%, ICC: 0.85 [0.72-0.92]), and $100 \% \mathrm{BW}$ (CV: $2.8 \%$, ICC: 0.89 [0.79-0.95]). There were no significant differences between test 1 and test 2 for any of the loads analyzed in the pretest and posttest. In addition, the magnitude of the differences between both tests was always trivial (Table 2).

\section{Discussion}

This study was designed to analyze whether the beneficial effects in explosive actions that followed a sudden ascent to altitude were maintained after a chronic exposure of 15-17 days to a moderate natural altitude. The results revealed significant increments in loaded squat jump performance when the tests were performed in hypoxia compared to normoxia in both pretest $(6.5 \%)$ and posttest $(4.5 \%)$ conditions. Given that the improvements caused by the physical factors should be similar in both tests, the $2.0 \%$ difference could be caused by physiological changes which occur during the exposure to the altitude. In addition, an overall increase in the load-velocity relationship after the altitude training period was also observed. These results suggest that altitude training could constitute a favorable stimulus for the development of explosive strength performance. However, the presence of a control group training in normoxia would have been recommended to examine further whether altitude training really has an additional benefit compared to training at the sea level.

Contrary to the deterioration in aerobic performance widely described at the altitude, some studies have shown that performance of non-aerobic dependant explosive actions improves at the altitude (Hahn and Gore, 2001; Kenney et al., 2012; Levine et al., 2008). In accordance with our results, an increase in the velocity at which a determined absolute load can be lifted following a sudden ascent to the altitude has also been described (Chirosa et al., 2006; Feriche et al., 2014). The improvements in movement velocity at a natural altitude may be caused by different factors such as: (a) reduced external resistance to the movement due to physical factors (Hahn and Gore, 2001; Kenney et al., 2012; Levine et al., 2008; Peronnet et al., 1991); (b) an incremented ability of the subject to produce force due to a stimulation of physiological factors, such as additional recruitment of fast twitch muscle fibers (Schoenfeld, 2013) or (c) a combination of physical and physiological factors.

Reduction in the external resistance to the movement has been proposed as one of the main reasons for the improvements in explosive actions performed at the altitude (Hahn and Gore, 2001; Kenney et al., 2012; Levine et al., 2008). Peronnet et al. (1991) indicated that running speed in sprint events (100 and $200 \mathrm{~m}$ ) was faster as the altitude increased (up to $4000 \mathrm{~m}$ ), due to the progressive reduction in air resistance without the detrimental effect of reducing energy availability. In addition, Feriche et al. (2014) compared the effects of acute exposure to a real (hypobaric hypoxia) or simulated altitude (normobaric hypoxia, 15.7\% inspired fraction of oxygen) on the force-velocity relationship during a bench press exercise in two groups of athletes from combat sports. While the 
group tested in hypobaric hypoxia showed an increase of $5.7 \%$ in the one-repetition maximum bench press, as well as a faster velocity for a given load compared to normobaric normoxia, no changes in any of these variables were observed in the group tested in normobaric hypoxia.

However, the slow velocities produced during loaded squat jumps suggest that it is unlikely that all differences can be explained by the physical factor alone (lower air resistance). In addition, if physical factors were the only cause, the same differences between hypoxia and normoxia would be expected in both tests. Nonetheless an increase of $6.5 \%$ in the overall load-velocity relationship considering the data from the pretest and an increase of $4.5 \%$ considering the data from the posttest were observed in hypobaric hypoxia. Given that air resistance was similar in both tests, the $2.0 \%$ difference could be explained by changes in physiological factors during the exposure to and training at the altitude.

Chronic hypoxia has been frequently associated with the loss of muscle mass (Deldicque and Francaux, 2013; Mizuno et al., 2008) and its functional capacity (Felici et al., 2001; Ferretti et al., 1990; Narici and Kayser, 1995; Raguso et al., 2004). However, most of these studies were conducted at high altitudes $>5000$ $\mathrm{m}$ asl), while altitude camps are usually held at moderate altitudes (1800-2500 m asl). Despite the fact that an ascent to moderate altitudes seems to improve the performance of explosive actions (Feriche et al., 2014), there is a lack of longitudinal studies analyzing the effects of altitude training camps on explosive muscle performance. In this context, the results of the present study revealed an overall increment of $4.0 \%$ in loaded squat jump velocity after the altitude training period when compared to normoxia tests, before and after the camp.

Wide variability in aerobic adaptations between individuals after altitude training is frequently reported (Friedmann et al., 2005). However, in the present study, only one swimmer in the pretest and another swimmer in the posttest developed higher velocity values during the tests conducted in normoxia. In addition, when considering the data from normoxia tests, $75 \%$ of the swimmers $(n=12)$ improved loaded squat jump performance by more than $1 \%$, whereas the changes in the remaining swimmers $(n=4)$ were \pm $1 \%$. Therefore, the altitude training camp worked well for most of the swimmers.

The improvement in loaded squat jump performance following the altitude training camp could have been caused by the occurrence of learning effects in the assessed exercise (Hopkins, 2000). However, based on the non significant differences and the trivial ES $(<0.10)$ between trials 1 and 2 in the pretest and posttest, we can affirm that there were no learning effects in our study sample. One would expect an increased performance in test 2 in comparison to test 1 (especially in the pretest) if the swimmers did not have the proper technique in the assessed exercise. Additionally, the test-retest reliability of the four loads studied was high (CV: $2.1 \%-3.1 \%$; ICC: $0.85-0.92)$. These results convince us that the change in performance was truly an effect of the altitude training and was not due to motor learning in the exercise assessed. However, a control group (training in normoxia) would have been necessary to examine further whether altitude training really has an additional benefit compared to training at the sea level.

In conclusion, the present findings indicate that loaded squat jump performance improves when the test is conducted at moderate natural altitudes compared to normoxic conditions. An overall increase in the loadvelocity relationship of $6.5 \%$ and $4.5 \%$ was observed after an acute (1-3 days) and chronic (1517 days) exposure to the moderate altitude, respectively. Physical and physiological factors arising from the reduction in the barometric pressure may be responsible for these changes. Given that the differences caused by the physical factors should be similar in both tests, the $2.0 \%$ difference observed between the two periods of exposure to the altitude could be caused by physiological changes. Although the main mechanisms of these improvements remain unclear, the overall training effect increment of $4.0 \%$ in loaded squat jump performance observed in normoxia following the altitude training camp suggests that such procedures constitute a favorable stimulus in the development of explosive strength. 


\section{Acknowledgements}

This study was supported by grants awarded by the Spanish Ministry of Science and Innovation (DEP2012-35774 \& DEP2015-64350-P MINECO/FEDER) and Ministry of Education, Culture and Sport (Predoctoral Grant FPU12/00360). The authors thank the swimmers and the coach (Albert Tubella) for their collaboration and commitment to this study.

\section{References}

Aeberli I, Erb A, Spliethoff K, Meier D, Götze O, Frühauf H, Fox M, Finlayson GS, Gassmann M, Berneis K, Maggiorini M, Langhans W, Lutz TA. Disturbed eating at high altitude: influence of food preferences, acute mountain sickness and satiation hormones. Eur J Nutr, 2013; 52: 625-635

Bazuelo-Ruiz B, Padial P, García-Ramos A, Morales-Artacho AJ, Miranda MT, Feriche B. Predicting maximal dynamic strength from the load-velocity relationship in squat exercise. J Strength Cond Res, 2015; 29: 1999-2005

Borg G, Ljunggren G, Ceci R. The increase of perceived exertion, aches and pain in the legs, heart rate and blood lactate during exercise on a bicycle ergometer. Eur J Appl Physiol Occup Physiol, 1985; 54: 343-349

Chirosa I, Feriche B, Calderón C, Martínez M, Braga R, Padial P. Ascent to moderate altitude improves force production. Arch Med Dep, 2006; 23: 101-108

Deldicque L, Francaux M. Acute vs chronic hypoxia: what are the consequences for skeletal muscle mass? Cell Mol Exerc Physiol, 2013; 2: e5

Di Prampero PE. Factors limiting maximal performance in humans. Eur J Appl Physiol, 2003; 90: 420-429

Etheridge T, Atherton PJ, Wilkinson D, Selby A, Rankin D, Webborn N, Smith K, Watt PW. Effects of hypoxia on muscle protein synthesis and anabolic signaling at rest and in response to acute resistance exercise. Am J Physiol Endocrinol Metab, 2011; 301: E697-E702

Felici F, Rosponi A, Sbriccoli P, Scarcia M, Bazzucchi I, Iannattone M. Effect of human exposure to altitude on muscle endurance during isometric contractions. Eur J Appl Physiol, 2001; 85: 507-512

Feriche B, García-Ramos A, Calderón-Soto C, Drobnic F, Bonitch-Góngora JG, Galilea PA, Padial P. Effect of acute exposure to moderate altitude on muscle power: hypobaric hypoxia vs. normobaric hypoxia. PLoS One, 2014; 9: e114072

Ferretti G, Hauser H, di Prampero PE. Maximal muscular power before and after exposure to chronic hypoxia. Int J Sports Med, 1990; 11: S31-S34

Friedmann B, Frese F, Menold E, Kauper F, Jost J, Bartsch P. Individual variation in the erythropoietic response to altitude training in elite junior swimmers. Br J Sports Med, 2005; 39: 148-153

Fulco CS, Friedlander AL, Muza SR, Rock PB, Robinson S, Lammi E, Baker-Fulco CJ, Lewis SF, Cymerman A. Energy intake deficit and physical performance at altitude. Aviat Space Environ Med, 2002; 73: 758765

Fulco CS, Rock PB, Cymerman A. Maximal and submaximal exercise performance at altitude. Aviat Space Environ Med, 1998; 69: 793-801

García-Ramos A, Štirn I, Padial P, Argüelles-Cienfuegos J, De la Fuente B, Strojnik V, Feriche B. Predicting vertical jump height from bar velocity. J Sports Sci Med, 2015; 14: 256-262

Hahn AG, Gore CJ. The effect of altitude on cycling performance: a challenge to traditional concepts. Sports Med, 2001; 31: 533-557

Hainsworth R, Drinkhill MJ, Rivera-Chira M. The autonomic nervous system at high altitude. Clin Auton Res, 2007; 17: 13-19

Hansen KT, Cronin JB, Pickering SL, Newton MJ. Does cluster loading enhance lower body power development in preseason preparation of elite rugby union players? J Strength Cond Res, 2011; 25: 2118-2126 
Hopkins W. Measures of reliability in sports medicine and science. Sports Med, 2000; 30: 1-15

Hopkins WG, Marshall SW, Batterham AM, Hanin J. Progressive statistics for studies in sports medicine and exercise science. Med Sci Sports Exerc, 2009; 41: 3-13

Hoppeler H, Desplanches D. Muscle structural modifications in hypoxia. Int J Sports Med, 1992; 13: S166-S168

Kenney WL, Wilmore J, Costill D. Physiology of Sport and Exercise. 5th ed. Champaign, IL: Human Kinetics; 2012

Levine BD, Stray-Gundersen J, Mehta RD. Scand J Med Sci Sports, 2008; 18: 76-84

Markovic G, Jaric S. Is vertical jump height a body-size independent measure of muscle power? J Sports Sci, 2007; 25: 1355-1363

Mizuno M, Savard GK, Areskog NH, Lundby C, Saltin B. Skeletal muscle adaptations to prolonged exposure to extreme altitude: a role of physical activity? High Alt Med Biol, 2008; 9: 311-317

Narici MV, Kayser B. Hypertrophic response of human skeletal muscle to strength training in hypoxia and normoxia. Eur J Appl Physiol Occup Physiol, 1995; 70: 213-219

Peronnet F, Thibault G, Cousineau DL. A theoretical analysis of the effect of altitude on running performance. J Appl Physiol, 1991; 70: 399-404

Raguso CA, Guinot SL, Janssens JP, Kayser B, Pichard C. Chronic hypoxia: common traits between chronic obstructive pulmonary disease and altitude. Curr Opin Clin Nutr Metab Care, 2004; 7: 411-417

Sánchez-Medina L, González-Badillo JJ. Velocity loss as an indicator of neuromuscular fatigue during resistance training. Med Sci Sports Exerc, 2011; 43: 1725-1734

Schoenfeld BJ. Potential mechanisms for a role of metabolic stress in hypertrophic adaptations to resistance training. Sports Med, 2013; 43: 179-194

Wehrlin JP, Hallen J. Linear decrease in $\mathrm{VO}_{2 \max }$ and performance with increasing altitude in endurance athletes. Eur J Appl Physiol, 2006; 96: 404-12

\section{Corresponding author:}

\section{Belén Feriche}

Faculty of Sport Sciences

University of Granada, Ctra. Alfacar, s/n, 18011, Granada (Spain)

Phone: (+34) 958244381

Fax: (+34) 958244369

E-mail: mbelen@ugr.es 